OPEN ACCESS

Edited by:

Lijuan Yuan,

Virginia Tech, United States

Reviewed by:

Anastasia N. Vlasova

The Ohio State University,

United States

Paul Licciardi,

Royal Children's Hospital, Australia

Viviana Parreño,

Instituto Nacional de Tecnología

Agropecuaria, Argentina

*Correspondence:

Jianguo $X u$

xujianguo@icdc.cn

${ }^{\dagger}$ These authors have contributed equally to this work

Specialty section:

This article was submitted to Nutritional Immunology,

a section of the journal

Frontiers in Nutrition

Received: 04 October 2021 Accepted: 16 November 2021 Published: 22 December 2021

Citation:

Xu J, Ren Z, Cao K, Li X, Yang J, Luo $X$, Zhu L, Wang $X$, Ding $L$,

Liang J, Jin D, Yuan T, Li L and Xu J (2021) Boosting Vaccine-Elicited

Respiratory Mucosal and Systemic COVID-19 Immunity in Mice With the Oral Lactobacillus plantarum.

Front. Nutr. 8:789242.

doi: 10.3389/fnut.2021.789242

\section{Boosting Vaccine-Elicited Respiratory Mucosal and Systemic COVID-19 Immunity in Mice With the Oral Lactobacillus plantarum}

\author{
Jianqing $\mathrm{Xu}^{1,2+}$, Zhihong Ren ${ }^{2,3 \dagger}$, Kangli Cao ${ }^{1 \dagger}$, Xianping $\mathrm{Li}^{3}$, Jing Yang ${ }^{3}$, Xuelian Luo ${ }^{3}$, \\ Lingyan Zhu ${ }^{2}$, Xiangwei Wang ${ }^{2}$, Longfei Ding ${ }^{2}$, Junrong Liang ${ }^{3}$, Dong Jin $^{3}$, Tingting Yuan ${ }^{2}$, \\ Lianfeng $\mathrm{Li}^{2}$ and Jianguo $\mathrm{Xu}^{3,4 *}$
}

'Zhongshan Hospital, Institutes of Biomedical Sciences, Shanghai Medical College, Fudan University, Shanghai, China, ${ }^{2}$ Shanghai Public Health Clinical Center, Fudan University, Shanghai, China, ${ }^{3}$ State Key Laboratory for Infectious Disease Prevention and Control, National Institute for Communicable Disease Control and Prevention, Chinese Center for Disease Control and Prevention, Research Units of Discovery of Unknown Bacteria and Function (2018 RU010), Chinese Academy of Medical Sciences, Beijing, China, ${ }^{4}$ Institute of Public Health, Nankai University, Tianjing, China

Boosting and prolonging SARS-CoV-2 vaccine-elicited immunity is paramount for containing the COVID-19 pandemic, which wanes substantially within months after vaccination. Here we demonstrate that the unique strain of probiotic Lactobacillus plantarum GUANKE (LPG) could promote SARS-CoV-2-specific immune responses in both effective and memory phases through enhancing interferon signaling and suppressing apoptotic and inflammatory pathways. Interestingly, oral LPG administration promoted SARS-CoV-2 neutralization antibodies even 6 months after immunization. Furthermore, when LPG was given immediately after SARS-CoV-2 vaccine inoculation, specific neutralization antibodies could be boosted $>8$-fold in bronchoalveolar lavage (BAL) and >2-fold in sera, T-cell responses were persistent and stable for a prolonged period both in BAL and the spleen. Transcriptional analyses showed that oral application of LPG mobilized immune responses in the mucosal and systemic compartments; in particular, gut-spleen and gut-lung immune axes were observed. These results suggest that LPG could be applied in combination with SARS-CoV-2 vaccines to boost and prolong both the effective and memory immune responses in mucosal and systemic compartments, thereby improving the efficacy of SARS-CoV-2 vaccination.

Keywords: COVID-19, vaccine, probiotics, adjuvant, gut-lung axis, memory immunity, Lactobacillus plantarum, accquired immunity

\section{INTRODUCTION}

The continued circulation of severe acute respiratory syndrome coronavirus 2 (SARS-CoV-2) has greatly impacted the global public health system and resulted in more than 249 million cases and over 5 million deaths. Hopes for returning to normal have largely depended on scaling-up inoculation with effective vaccines that are essential to prevent further morbidity and mortality. To date, there are 194 and 128 COVID-19 vaccine candidates in preclinical or preclinical development, 
respectively, and 21 vaccines are currently been offered to the general population (1). The World Health Organization estimated that at least $70 \%$ of the world's population will need to be vaccinated to stop the pandemic. The persistent evolution of SARS-CoV-2 led to the emergence of new contagious variants that fueled the spread of disease. It remains unknown how effective the vaccines are against these mutations and how long they will protect against viral transmission (2). The antibody responses following natural infection wane substantially within months (3), so it is possible that immunity generated by vaccines might only last for months. Thus, containing the pandemic will require strategies that boost and prolong vaccine-elicited immune responses.

Probiotics confer a health benefit to the host when administered in adequate amounts (4). We hypothesize that oral administration of certain specific strain of probiotics could enhance and probably prolong COVID-19 vaccine-elicited immune responses through a mechanism of regulating both innate and acquired immune responses. Here we demonstrate that a unique strain of probiotic Lactobacillus plantarum GUANKE (LPG) is capable of promoting SARS-CoV-2 specific immune responses in both effective and memory phases through enhancing interferon (IFN) signaling and suppressing apoptotic and inflammatory pathways.

\section{MATERIALS AND METHODS}

\section{Ethics Statement and Animals}

The aim of this study was to determine the effect of LPG on the immune response to the SARS-CoV-2 vaccine in mice. All animal-related procedures were conducted according to the protocol approved by the Institutional Animal Care and Use Committee (IACUC) of Shanghai Public Health Clinical Center (Shanghai, China). Specific pathogen-free (SPF) female ICR and $\mathrm{BALB} / \mathrm{c}$ mice were purchased from Shanghai Jihui Biological Co., Ltd and housed in the animal facility at Shanghai Public Health Clinical Center in environmentally controlled cages with a 12 -hlight/-dark cycle under SPF conditions. Mice were provided with free access to water and diets.

\section{Mice Immunization}

The aim of the first experiment was to determine the effect of oral LPG administration on mouse humoral immune responses to COVID-19 when given 6 months after immunization. All ICR mice $(n=10)$ received intramuscular vaccine injections at weeks 0 and 4 post priming, and sera were collected between weeks 5 and 24 post priming for antibody assessment by enzyme-linked immunosorbent assays (ELISAs) and pseudovirus inhibition assays. At 24 weeks post vaccination, the mice were given water containing $1 \mathrm{~g} / \mathrm{L}$ of ampicillin for 5 days to prevent colonization resistance (5), then they were randomly divided into two groups that received oral administration of either $200 \mu \mathrm{L}$ of LPG $(5 \times$ $10^{9} \mathrm{CFU}, n=6$ ) or phosphate-buffered saline (PBS, $n=4$ ) once daily for 3 days. The antibody titers measured immediately before ampicillin administration were set as the baseline to be compared with measurements taken at various time points post LPG or PBS administration. Sera were collected on day $7,14,28$, and 42 post oral administration of LPG or PBS for receptor-binding domain (RBD) binding antibody assessment by ELISA and neutralization antibody ( $\mathrm{nAb}$ ) quantification by pseudovirus inhibition assay.

The second experiment was conducted to determine the effect of oral LPG administration on humoral and cellular immune responses in mice when given immediately after immunization. All ICR mice were primed with an intramuscular injection of $100 \mu \mathrm{g}$ of DNA-S at week 0 . At week 12 after priming, the mice were given drinking water containing $1 \mathrm{~g} / \mathrm{L}$ ampicillin for 5 days then boosted with $1 \times 10^{11}$ viral particles (vp) of AdC68$\mathrm{CoV} / \mathrm{Flu}$ via both intramuscular and intranasal routes. The mice were then randomly divided into two groups ( $n=9$ /group) for oral administration of either $200 \mu \mathrm{L}$ of LPG $\left(5 \times 10^{9} \mathrm{CFU}\right)$ or PBS once daily for 3 days. The antibody titers measured immediately before ampicillin administration were set as the baseline to be compared with measurements taken at various time points post LPG or PBS administration. The mice $(n=$ 3/group) were sacrificed on day 3, 7, 14 post administration of LPG or PBS. Sera and bronchoalveolar lavage (BAL) were collected on baseline and day 7 post administration of LPG or PBS for RBD binding antibody and $\mathrm{nAb}$ assessment. To assess RBD-specific $T$ cell responses, splenocytes and BAL cells were isolated on day 3, 7, and 14 post oral administration of LPG or PBS and in vitro stimulated with 13 peptide pools (15-mer with 11 overlapped amino acids) covering the entire RBD sequence. The resulting IFN $-\gamma$ secreting cells were quantified by ELISpot. Interleukin (IL)-6 and tumor necrosis factor (TNF)- $\alpha$ in BAL from mice sacrificed on day 3, 7, and 14 were quantified using Th1/2/17 Assay Kit (Cat\#560485, BD Bioscience, Franklin Lakes, NJ, USA).

\section{Oral LPG Administration of Mice for Transcriptional Analyses}

To examine how LPG imprints the immune system. Two groups of ICR mice (20 mice per group) were given $1 \mathrm{~g} / \mathrm{L}$ of ampicillin for 5 days followed by oral administration of $200 \mu \mathrm{L}$ of LPG $\left(5 \times 10^{9} \mathrm{CFU}\right)$ or PBS once daily for 3 days. Different tissues including spleen, mediastinal lymph node (LN), mesenteric LN, small intestine, and colon tissues were collected and stored in TRIzol at different time points after oral administration for RNAsequencing.

\section{Bacterial Culture}

LPG isolated from the feces of healthy people was routinely grown in Man-Rogosa-Sharpe medium in a $37^{\circ} \mathrm{C}$ carbon dioxide incubator (Forma CO2, Thermo Fisher Scientific, Waltham, MA, USA). The logarithmic phase of LPG was washed and resuspended with sterile PBS for oral inoculation of mice.

\section{Cell Lines and Viruses}

The human cell lines used in this study included HEK 293A and HEK 293T. All cell lines were purchased from American Type Culture Collection (Manassas, VA, USA) and maintained in complete Dulbecco's modified Eagle's medium supplemented with $10 \%$ fetal bovine serum (FBS) and $1 \%$ penicillin-streptomycin (PS) (D10) at $37^{\circ} \mathrm{C}$ in a $5 \% \mathrm{CO}_{2}$ incubator. The generation of wild-type SARS-CoV-2 pseudovirus 
was described in our previous publications $(6,7)$. In brief, HEK 293T cells were co-transfected with pNL4-3.Luc.R-E(cat\#3418, NIH AIDS Reagent Program) and pcDNA3 plasmid encoding SARS-CoV2-S using TurboFect reagent (cat\#R0531, Thermo Fisher Scientific). At $24 \mathrm{~h}$ post transfection, the culture medium was refreshed with D10 medium for an additional 48$\mathrm{h}$ incubation. The pseudovirus-containing supernatants were harvested by centrifugation then filtered through $0.45-\mu \mathrm{m}$ filters and stored at $-80^{\circ} \mathrm{C}$ as single-use aliquots. For each pseudovirus stock, a titration assay was performed using an aliquot to determine the dilution needed for the neutralization assay.

\section{DNA Vaccine Construction}

The DNA-S is a DNA vaccine candidate for which the codonoptimized DNA sequences encoding SARS-CoV-2 spike (S) protein (YP_009724390.1) were synthesized (Generay Biotech Co., Ltd., Shanghai, China), and cloned into the pcDNA3.1 vector to generate the pcDNA3.1-S plasmid.

\section{Construction, Rescue, Propagation, and Purification of Recombinant Chimpanzee Adenoviruses}

The AdC68-CoV/Flu is a chimpanzee adenoviral vaccine with a hemagglutinin stalk derived from influenza A/Shanghai/4664T/2013 strain and the RBD of wild-type SARS-CoV-2, which were synthesized and cloned into the E1 site of the recombinant AdC68 vector. The recombinant AdC68 is different from the wild-type AdC68 in that both the E1 and E3 genes are deleted. The E1 locus is essential for viral replication, so recombinant AdC68 viruses can only be produced in cell lines engineered to supply the E1 protein, such as HEK 293A. HEK 293A cells were seeded in 6-well plates for $24 \mathrm{~h}$ then were transfected with a PacI linearized corresponding AdC68 construct using Lipofectamine 2000 following the manufacturer's instruction (Invitrogen, Carlsbad, CA, USA). The rescued viruses were propagated in HEK 293A cells and purified using $\mathrm{CsCl}$ gradient ultracentrifugation in combination with desalting with Bio-Gel P-6 DG Media (Bio-Rad, Hercules, CA, USA).

\section{RBD Binding Antibody Detection Using ELISA}

RBD binding antibody titers in serum and BAL samples were measured by ELISA. In brief, sera and BAL samples were heatinactivated at $56^{\circ} \mathrm{C}$ for $30 \mathrm{~min}$ before use. Next, 96-well ELISA plates were coated with $100 \mu \mathrm{L}$ of $1 \mathrm{mg} / \mathrm{mL}$ recombinant SARSCoV-2 RBD protein (Z03483-1, Genscript, Piscataway, NJ, USA) at $4{ }^{\circ} \mathrm{C}$ overnight. After washing the plates with $300 \mu \mathrm{L}$ PBS containing $0.5 \%$ Tween-20 (PBS-T) and blocking with $200 \mu \mathrm{L}$ $5 \%$ non-fat milk in PBST (PBST/5\% milk) for $2 \mathrm{~h}$ at room temperature (RT), a 2-fold dilution series (generally starting from 1:100) of mouse serum and BAL samples were added, followed by 3 -h incubation at RT. For immunoglobulin G (IgG) measurement, a 1:5,000 dilution of horseradish peroxidase (HRP)-conjugated goat anti-mouse IgG (ZB-5305, Zsbio, Beijing, China) was applied in $100 \mu \mathrm{L}$ PBST/5\% milk. After $1 \mathrm{~h}$ incubation at RT, the plates were extensively washed with $300 \mu \mathrm{L}$
PBS-T before addition of the substrate OPD (one SIGMAFAST OPD tablet [SLCC0308, Sigma, St. Louis, MO, USA] in $20 \mathrm{~mL}$ of deionized water). The reactions lasted for $5 \mathrm{~min}$ at RT and were terminated by adding $1 \mathrm{M} \mathrm{H}_{2} \mathrm{SO}_{4}$, followed by reading at OD492 with a Synergy Microplate Reader (Bio-Tek, Winooski, VT, USA). ELISA endpoint titers were defined as the highest dilution that yielded an absorbance that was 2-fold greater than the background value.

\section{Total IgG ELISA}

Total serum IgG levels were measured using the IgG (Total) Mouse Uncoated ELISA kit (Cat\# 88-50400, Thermo Fisher Scientific). Each sample was diluted 500-fold in assay buffer and run in duplicate with Southern Biotech TMB Stop Solution (Cat\#0412-01) as the stop solution. Optical density values were measured at $450 \mathrm{~nm}$ and $570 \mathrm{~nm}$ on a VersaMax plate reader (Molecular Devices, Sunnyvale, CA, USA) and corrected by subtracting the measurement at $570 \mathrm{~nm}$ from the measurement at $450 \mathrm{~nm}$. A four-parameter standard curve was used to calculate sample concentration values.

\section{Pseudovirus Neutralization Assay}

The protocol for SARS-CoV-2 pseudovirus generation was described previously (7). For assessment of serum neutralizing activity against SARS-CoV-2, serial $50-\mu \mathrm{L}$ dilutions of heatinactivated serum were made with D10 medium and mixed with equal volumes of diluted pseudovirus. After incubation at $37^{\circ} \mathrm{C}$ for $1 \mathrm{~h}$, the serum-pseudovirus mixtures were transferred to a 96-well plate containing hACE2-293T cells, which were pre-seeded at $2 \times 10^{4}$ cells per well and allowed to grow for $12 \mathrm{~h}$. The plates were incubated for $48 \mathrm{~h}$ at $37^{\circ} \mathrm{C}$ in the presence of $5 \% \mathrm{CO}_{2}$, and luciferase activities were then measured using Bright-Glo ${ }^{\text {TM }}$ Luciferase Assay System (Promega, Madison, WI, USA) on a luminometer (Promega GloMax 96). The neutralizing (50\% inhibitory doses) titers were derived from the highest dilution resulting in a $50 \%$ reduction in relative light units (RLUs) relative to virus control wells after background (RLUs of no virus wells) subtraction.

\section{IFN- $\gamma$ Based ELISpot Assay}

$\mathrm{T}$ cell responses were analyzed by using single-cell suspension of splenocytes and BAL by mouse IFN- $\gamma$ ELISpot assay set (Cat\#551083, BD Bioscience) following the manufacturer's protocol. In brief, 96-well ELISpot plates were pre-coated with anti-mouse IFN- $\gamma$ antibodies $(5 \mu \mathrm{g} / \mathrm{mL}, 100 \mu \mathrm{L})$ overnight at $4^{\circ} \mathrm{C}$. After rinsing with RPMI-1640 medium (10-040-CVR, Corning, Corning, NY, USA) containing 10\% FBS (BI, 04-0011acs) and 1\% PS (30-002-CI, Corning) (R10), plates were blocked with R10 for $2 \mathrm{~h}$ at RT before adding to each well $2 \times 10^{5}$ of splenocytes or $2 \times 10^{4}$ of BAL cells, followed by stimulation with the indicated 15-mer peptide(s) or pool(s). The peptides used for the stimulation work were synthesized in ChinaPeptides Co., Ltd. Each assay was performed in duplicate. Peptide stimulation lasted for $20 \mathrm{~h}$ after plates were placed in a humidified $5 \% \mathrm{CO}_{2}$ incubator at $37^{\circ} \mathrm{C}$. The plates were washed before biotinylated anti-mouse IFN- $\gamma$ antibody $(2 \mu \mathrm{g} / \mathrm{mL}, 100 \mu \mathrm{L})$ was added for 2 h RT incubation. Next, streptavidin-conjugated HRP was added 
at 1:100 dilution and incubated at RT for $1 \mathrm{~h}$. The plates were washed again and subjected to spot development with AEC substrate reagent (Cat\#551951, BD Bioscience). The reaction was stopped by water rinsing, and the plates subsequently allowed to dry for $24 \mathrm{~h}$ in the dark. Plate images were captured with a Biospot plate reader (ChampSpot III, Beijing SageCreation Science Co., Ltd., Beijing, China) and analyzed for spot-forming cell counts. Quality control was implemented on the automatic counting data by removing pseudo-spots, such as those resulted from pollutants and filaments and other residues. R10 was used as a negative control and all the data were adjusted by subtracting the value of negative control, 50 SFCs/million was considered as cut off value.

\section{BAL Cytokines}

Flow cytometry assessments of serum levels of IL- 6 and TNF- $\alpha$ were carried out using the BD Mouse Cytometric Bead Array kit for Th1/Th2/Th17 cytokine panel (Cat\#560485, BD Bioscience).

\section{RNA-Sequencing and Quantitative Real-Time Polymerase Chain Reaction}

RNA-sequencing was performed to examine how LPG imprints the immune system. Total RNA of different tissues were extracted using the TRIzol agent following the manufacturer's instruction, and total RNA quality was determined using the Agilent 2100 (Agilent Technologies, Santa Clara, CA, USA). A total of $5 \mathrm{ng}$ purified RNA was used for library construction with an Illumina ${ }^{\circledR}$ TruSeq ${ }^{\circledR}$ RNA Sample Preparation Kit v2 (Cat\#RS-122-2001, Illumina, San Diego, CA, USA), and the library was sequenced on Illumina HiSeqTM2500 by Shanghai South Gene Technology Co., Ltd. (Shanghai, China). The clean data were obtained by removing adapter-containing reads and low-quality reads by FASTX-Toolkit (http://hannonlab.cshl.edu/ fastx_toolkit/). These clean reads were mapped to the reference genes using Bowtie2 software and to the reference genome using HISAT software. Counts were converted to fragments per kilobase of exon per million mapped fragments (FPKM) values for normalization. Gene expression data were subjected to set enrichment analysis using gene set enrichment analysis (GSEA) and hierarchical cluster analysis with Multiexperiment Viewer ( $\mathrm{MeV}$ 4.9).

\section{Statistical Analysis}

All continuous variables are presented as means \pm standard deviation. Univariate analysis of variance was used to compare the means of different groups, and Bonferroni or Dunnett tests, if appropriate, were used for multiple comparisons if their variance homogeneity was assumed. Otherwise, Kruskal-Wallis tests were used followed by pairwise comparisons if appropriate. Statistical analysis was performed using GraphPad Prism v.8.0 (GraphPad Inc., San Diego, CA, USA). Comparisons of antibody titer levels between the two groups were analyzed using unpaired $t$-tests or Mann-Whitney tests.

\section{RESULTS}

\section{LPG Promotes and Prolongs SARS-CoV-2 Specific Memory Antibody Responses in Mice}

In 1998, we accidentally found that oral LPG administration could boost the specific humoral immune responses against Shigella dysenteriae in vaccinated BALB/c mice (8). The unique strain of LPG we used is phylogenetically close to $L$. plantarum J26 with 12 additional genes based on whole genome sequences analyses of 611 strains available in public database (9) (Supplementary Figure 1). In this study, we sought to determine if LPG could also improve immune responses in a SARS-CoV-2 vaccinated model. A group of ICR mice were inoculated with the DNA vaccine at weeks 0 and 4 (Figure 1A). The results of the sera RBD binding antibody titers (Figure 1B) and $\mathrm{nAb}$ titers (Figure 1C) from weeks 5 to 24 showed that both antibody titers maintained their peak levels from weeks 5 to 8 and then decreased gradually over the 24-week follow up. Then these mice were orally administered with PBS or LPG and observed for 42 days. As expected, both groups had similar levels of $\mathrm{RBD}$-specific antibodies and $\mathrm{nAbs}$ at baseline. The binding antibodies and nAbs gradually decreased in the PBS group (Figures 1D,F, filled circles), consequently, the index (ratios of titers of antibody at indicated time points to their counterparts at baseline, reflecting the adjusted values after the removal of differences at baseline) values decreased below one and further subsided over time (Figures 1E,G, filled circles). In contrast, the binding antibodies stabilized in the LPG group for at least 28 days (Figures 1D,E, open circles), while nAbs progressively elevated. Accordingly, the differences (folds between LPG and PBS groups in Figure 1F) also increased from 1.15 to 3.9 fold, which were further evidenced by the "index" values and were significantly different on day 28 and $42(P<0.01$; Figure 1G). To determine whether $\mathrm{nAb}$ enhancement was due to the overall increase of host total antibody responses, we compared total IgG titers and found no significant difference between the PBS and LPG groups (Supplementary Figures 2A,B). Together, these results demonstrate that LPG was able to boost and prolong memory antibody responses in the absence of vaccine or immunogen.

\section{Oral LPG Administration Immediately After SARS-CoV-2 Vaccination Significantly Boosts Both Effective Humoral and Cellular Responses Against COVID-19 in Mice}

We next sought to determine whether LPG was capable of boosting vaccine-induced effective immune responses in the SARS-CoV-2 vaccination model. ICR mice were primed and boosted with vaccines followed by oral administration of LPG or PBS as described in detail in the Methods (Figure 2A). As shown in Figure 2B, serum RBD binding antibody titers at baseline (before ampicillin treatment) were comparable in the PBS and LPG groups with a geometric mean titer (GMT) of 9,406 (left panel). On day 7 after boost, the titers increased to GMT 129,016 in the PBS group, and a further 6.3-fold increase to GMT 819,200 was observed in the LPG group ( $P$ 


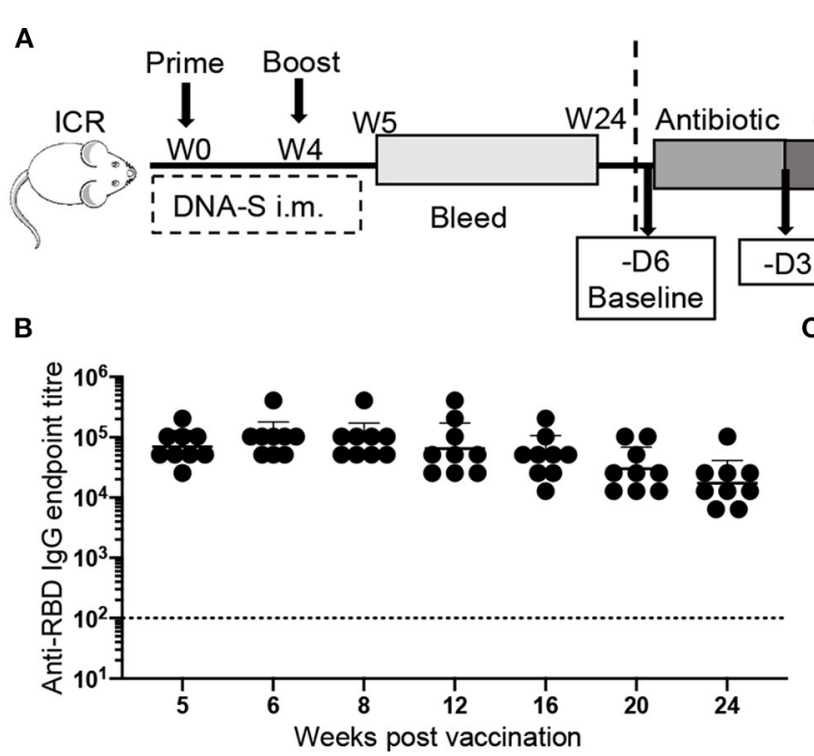

D
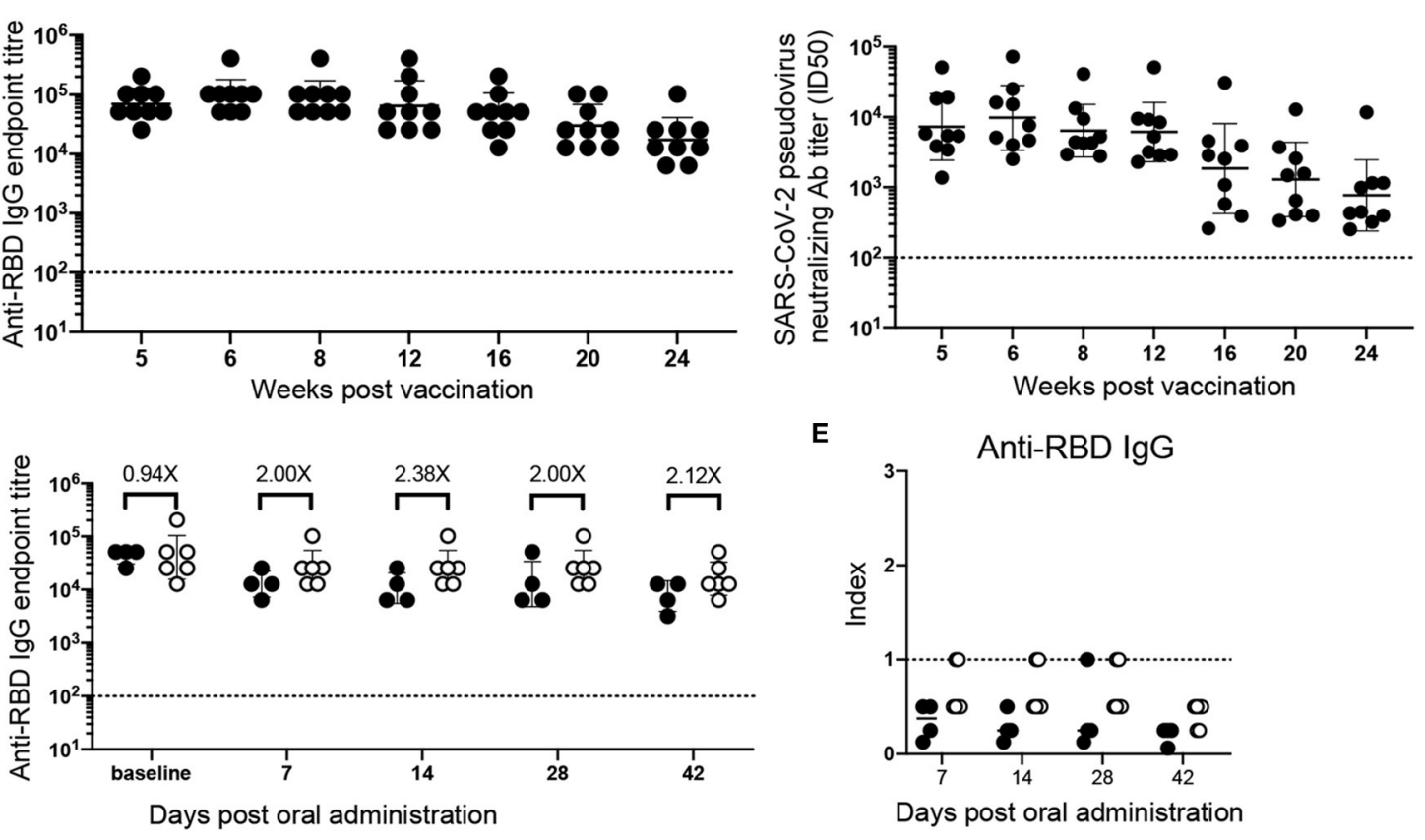

E

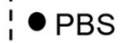

O IPG
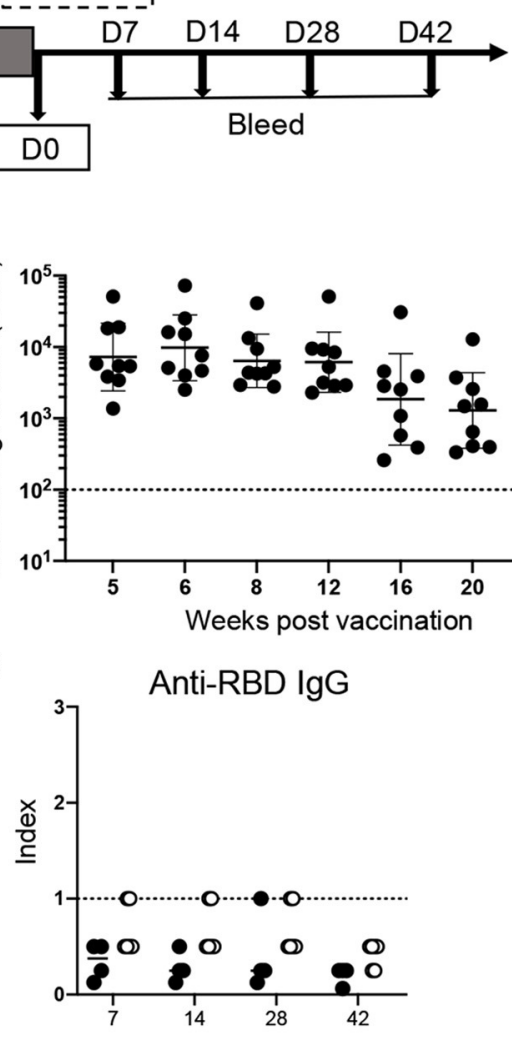

Days post oral administration

F

G
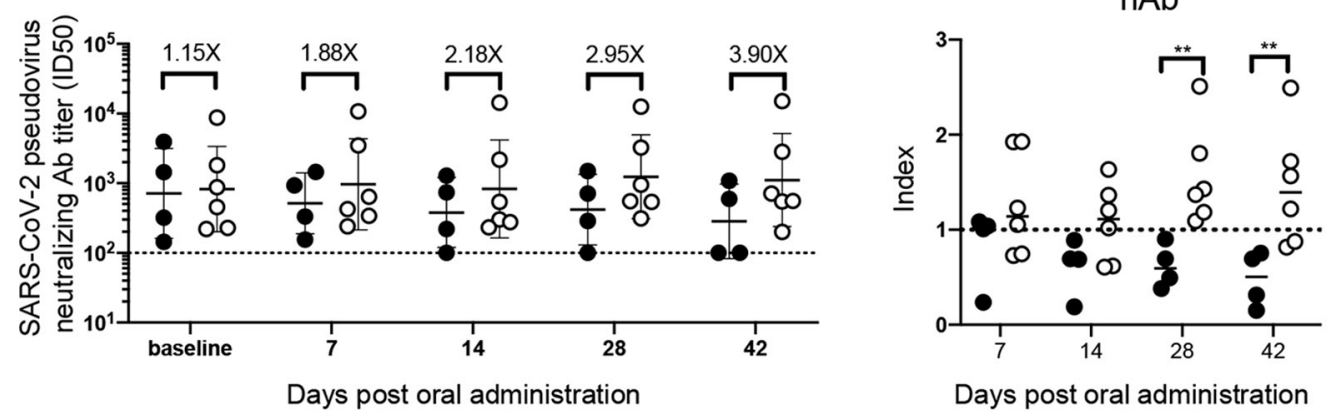

Days post oral administration

FIGURE 1 | Oral LPG administration 6 months after immunization improved humoral immune responses in mice. Experimental schedule (A): ICR mice were vaccinated with DNA vaccine at weeks 0 and 4, their binding (B) and neutralization (C) antibody responses were monitored by ELISA and pseudovirus inhibition assays respectively, from 5 weeks to 24, these mice were then orally administrated with PBS or LPG and observed for 42 days. The antibody titers immediately before antibiotic treatment were set as baseline. Sera RBD binding (D) and neutralization (F) antibodies on day 7, 14, 28, and 42 post administration of LPG or PBS were assessed by ELISA and pseudovirus inhibition assay, respectively. "Index" on the Y-axis represents the ratios of titers of binding antibodies (E) and neutralization (G) at indicated time points on the X-axis to their counterparts at baseline and reflects the adjusted relative values after the removal of baseline differences. D: Day; W: Week. Titer data are presented as geometric mean titer \pm geometric standard deviation. Mann-Whitney tests were performed to analyze differences between experimental groups ${ }^{\star \star} P<0.01$

$<0.001$; right panel). Similarly, a 2.3-fold promotion of $\mathrm{nAb}$ responses was observed in the LPG group compared with the PBS group, although significance was not reached (Figure 2C, right panel). We further assessed binding antibody and $\mathrm{nAb}$ titers in BAL collected on day 7 post oral administration.
Binding antibody titers in the LPG group (GMT $=3,225)$ were $\sim 10$-fold higher than in the PBS group $(\mathrm{GMT}=320$; Figure 2D), as were $\mathrm{nAb}$ titers in the LPG group (GMT = $241)$ vs. the PBS group (GMT $=27$; Figure $2 E)$. We next examined $\mathrm{RBD}$-specific $\mathrm{T}$ cell responses in the spleen and 
in BAL isolated from immunized mice on day 3, 7, and 14 post oral LPG administration by ELISpot assay, which captures IFN- $\gamma$ after stimulation with pools of RBD-derived peptides. A total of $13 \mathrm{RBD}$ peptide pools were used to fully cover the RBD sequence, each comprising five peptides (15-amino acid) that overlap by 11 amino acids. Although the boost vaccination induced similar T-cell responses in the LPG and PBS groups (data shown as day 3), they rapidly decreased in the PBS group and remained relatively stable in the LPG group (Figures 2F,G and Supplementary Figures 3A,B). Collectively, these results suggest that oral LPG administration boosts effective humoral and cellular responses against SARS-CoV-2 in vaccinated mice.

\section{Oral LPG Regulates Immune Responses Through Gut-Lung and Gut-Spleen Immune Axes}

To examine how LPG imprints the immune system, we collected spleen, mediastinal LN, mesenteric LN, small intestine, and colon tissues to define their transcriptional landscapes with RNA-sequencing after oral LPG administration (Figure 3A). Surprisingly, three similar transcript profiles were identified among mesenteric LN, mediastinal LN, and spleen, including downregulated TNF- $\alpha$ (a representative marker of inflammation), apoptosis, and enhanced IFN responses in the LPG group compared to the PBS group (Figure 3B), suggesting that oral LPG can exert effects in both the mucosal and systemic immune systems to promote immune cell activation and differentiation. Interestingly, LPG actively down-regulated the TNF- $\alpha$ pathway, as evidenced by reduced expression of AP-1 transcription factor family members (FOS, FOSB, JUN, JUNB) (10) (Figure 3B, upper panel). It also dampened apoptosis pathways, shown by decreased expression of the apoptotic genes BCL2L11, BTG2, and BTG3 $(11,12)$ (Figure 3B, middle panel). This down-regulation began on day 0 and peaked on day 1 for the mesenteric LN and spleen and on day 3 for the mediastinal LN, indicating that LPG almost simultaneously exerted its effects on the mesenteric LN and spleen with a slight delay for the mediastinal LN. However, this downregulation was not observed in small intestine and colon tissues, suggesting that this mechanism largely functions on immune cells rather than digestive tissues. To further corroborate this observation, we quantified IL- 6 and TNF- $\alpha$ in BAL from mice sacrificed on day 3,7 , and 14 . A $\sim 20$-fold reduction in IL-6 and 3-8-fold decrease in TNF- $\alpha$ were observed on day 3 and 7 , respectively, in the LPG group compared with the PBS group (Figure 3C).

In contrast to down-regulation of TNF- $\alpha$ and apoptosis signaling, LPG up-regulated the IFN response pathway as manifested by increased expression of IFN-regulatory factor 7 (IRF7) and IFN-stimulated genes IFITM1/2/3 (13) in the small intestine, spleen, mesenteric LN, and less for the mediastinal LN, but not for colon tissue (Figure 3B, down panel). This up-regulation was triggered in both digestive tissues and lymph tissues with the exception of colon tissue. These results indicate that oral LPG administration can affect the lung immune response through the gut-lung and gut-spleen immune axes.

\section{Oral LPG Administration Promotes T and B Cell Responses Through Costimulatory and Survival Pathways}

We next examined how the above-described immune microenvironment influences adaptive immune responses. A heatmap with hierarchical clustering analyses of RNAsequencing on the $\mathrm{T}$ cell- and $\mathrm{B}$ cell-associated genes showed different transcriptional signatures in lymphoid and intestine tissues (Supplementary Figure 4), suggesting that the datasets are reliable. As shown in Figure 3D, a number of genes in the $\mathrm{B}$-cell response (BCR) signaling pathway were up-regulated in the spleen and mesenteric LN on day 0 in the LPG group. Delayed up-regulation of those genes was also observed on day 7 in the mediastinal LNs. In addition, the co-stimulatory molecules CD86, ICOSL, and TNFR-associated factor (TRAF) family members (14) for B cell activation, proliferation, and differentiation were enhanced in the LPG group. Furthermore, several genes associated with $\mathrm{B}$ cell functions such as $A K T 1 / 2$, $A I C D A$, and $E B I 3$ were upregulated in the LPG group, indicating that LPG improved B cell activation and their differentiation to memory B cells (Figure 3D, signaling panel). For transcriptional factors, the $\mathrm{T}_{\mathrm{fh}}$ master transcriptional factor $B C L-6$ and those responsible for $\mathrm{B}$ cell differentiation (EBF1 and PAX5) (15) and the activation and switch to plasma cells (XBP1) (16) peaked on day 0 in the spleen and varied in LNs in the LPG group (Figure 3D, transcription panel). In general, the entire set of genes for B cell activation, proliferation, memory, and differentiation was up-regulated early in the LPG group, which corroborates our observation of improved SARS-CoV-2 humoral responses.

We further examined the transcriptional signatures of $\mathrm{T}$ cell subsets. We found that molecules of the CD28B7, TNFSF/TNFRSF, and TRAF families were upregulated in the LPG group (Figure 3E, signaling panel), supporting our observation that LPG enhanced T cell responses to SARS-COV-2 (Figures 2F,G and Supplementary Figures 3A,B). Accordingly, IL genes and those of the down-stream JAK-STAT pathway were up-regulated in LPG group, including $I L-2 R, I L-7 R, I L$ $15 R$, and $S T A T 1 / 3 / 5$ (Figure 3E, signaling panel, middle). These data indicate that LPG could facilitate T cell survival and their differentiation into memory $\mathrm{T}$ cells. CCR7, CXCR3, CXCR5, and the $S 1 P R$ family are associated with cell recruitment and antigen collection, and the decrease of CCR7 and increase of S1PR family members in the spleen of the LPG group promoted $\mathrm{T}$ cell emigration from lymph tissues (Figure 3E, signaling panel, bottom). We further observed up-regulation of the master transcriptional factors such as EOMES, TBX21, GATA3, and BCL6 in different CD4+ subsets, indicating a more active $\mathrm{T}$ cell response in the LPG group (17). We also found increased expression of BATF, PRDM1, and IRF family genes involved in sustaining virus-specific CD8+T cell function (18) (Figure 3E, transcriptional panel). The decreases in $I L-17 A$ and $I L-17 B$ indicate down-regulation of inflammation (19), while 
A

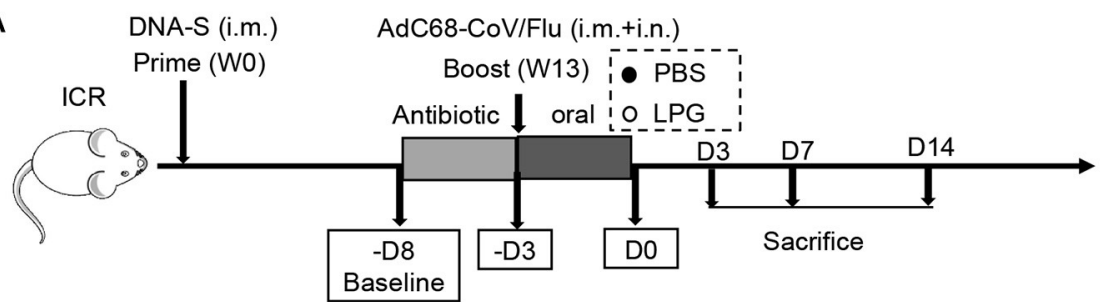

B
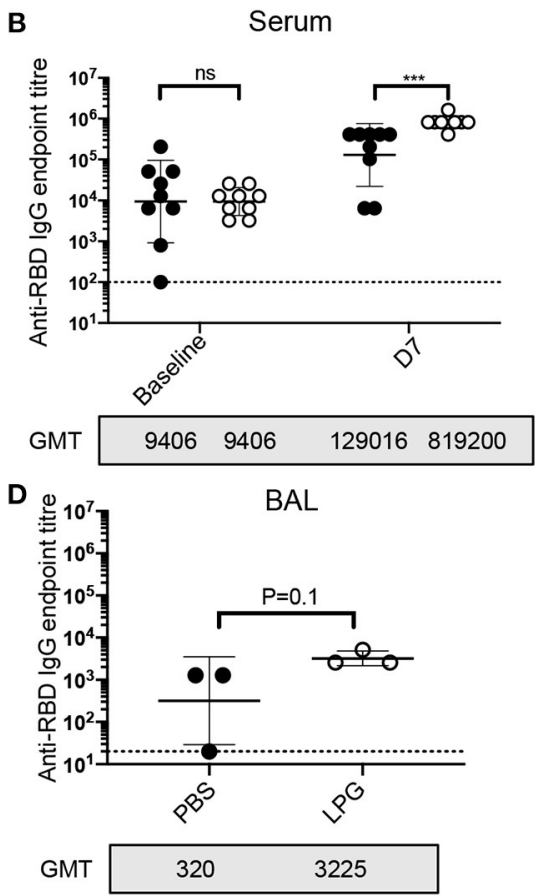

$\mathbf{F}$

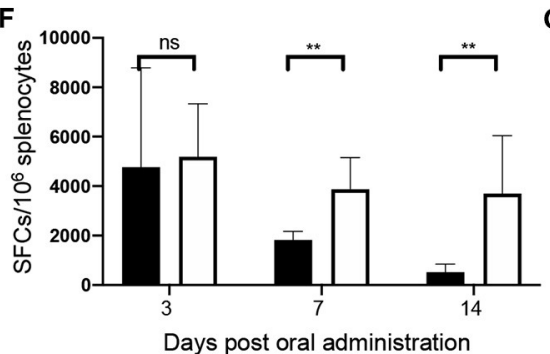

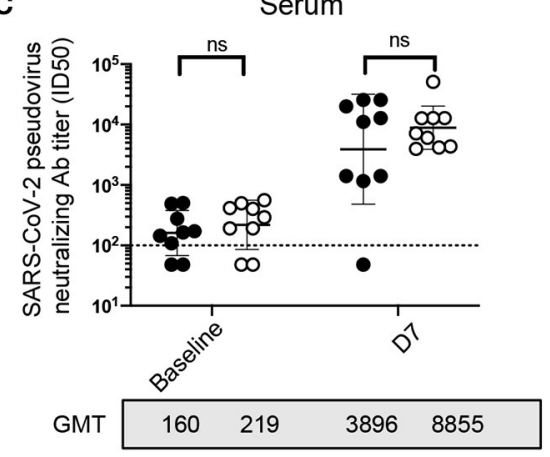

E

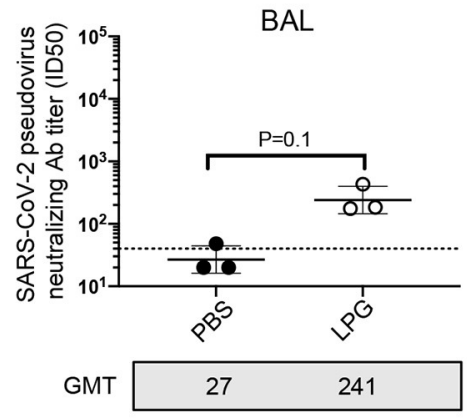

G

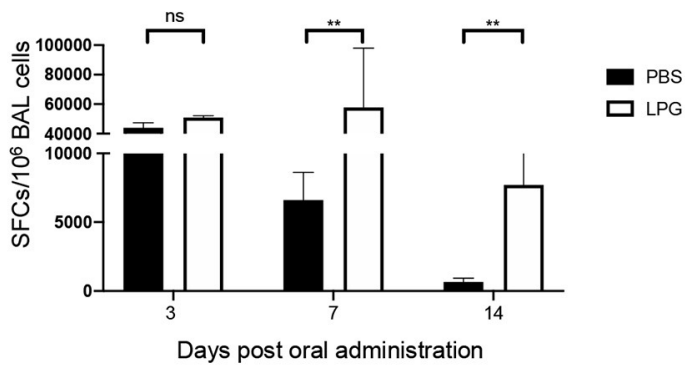

FIGURE 2 | Oral LPG administration immediately after immunization improved humoral and cellular immune responses in mice. Experimental schedule (A). ICR mice were primed and boosted with vaccines followed by oral administration of LPG or PBS as described in the Methods. Serum antibody responses were assessed at baseline (immediately before antibiotic treatment) and day 7 post intragastric administration (D7) for RBD-specific binding antibodies by ELISA (B) and pseudovirus neutralization assays (C). BAL antibody responses were assessed on day 7 post intragastric administration for RBD-specific binding antibodies by ELISA (D) and pseudovirus neutralization assays (E). Assessments of RBD-specific T cell responses. T-cell responses in splenocytes (F) and BAL cells (G) were determined on day 3, 7, and 14 post intragastric administration. BAL: Bronchoalveolar lavage; D: Day; W: Week. Titer data are presented as geometric mean titer \pm geometric standard deviation; ELISpot counts were expressed as mean \pm s.e.m. Mann-Whitney tests were performed to analyze differences between experimental groups. ${ }^{\star \star} P<0.01$,

${ }^{\star \star \star} P<0.001$, and ns, not significant.

increases in PRF1, GZMA, and GZMB suggest vigorous cytotoxic function (20) (Figure 3E, cytokine panel) in the LPG group. Interestingly, all these observations manifested in the spleen and mesenteric LN, but not in the mediastinal LN, which may be due to the delayed response for the mediastinal $\mathrm{LN}$ as shown in Figure 3E.

\section{DISCUSSION}

It has been reported that antibody titers in subjects who have recovered from SARS-CoV-2 infection rapidly wanes over time (21). This raises concerns of how long vaccine-elicited immune responses will last, which will dictate when boost inoculation 


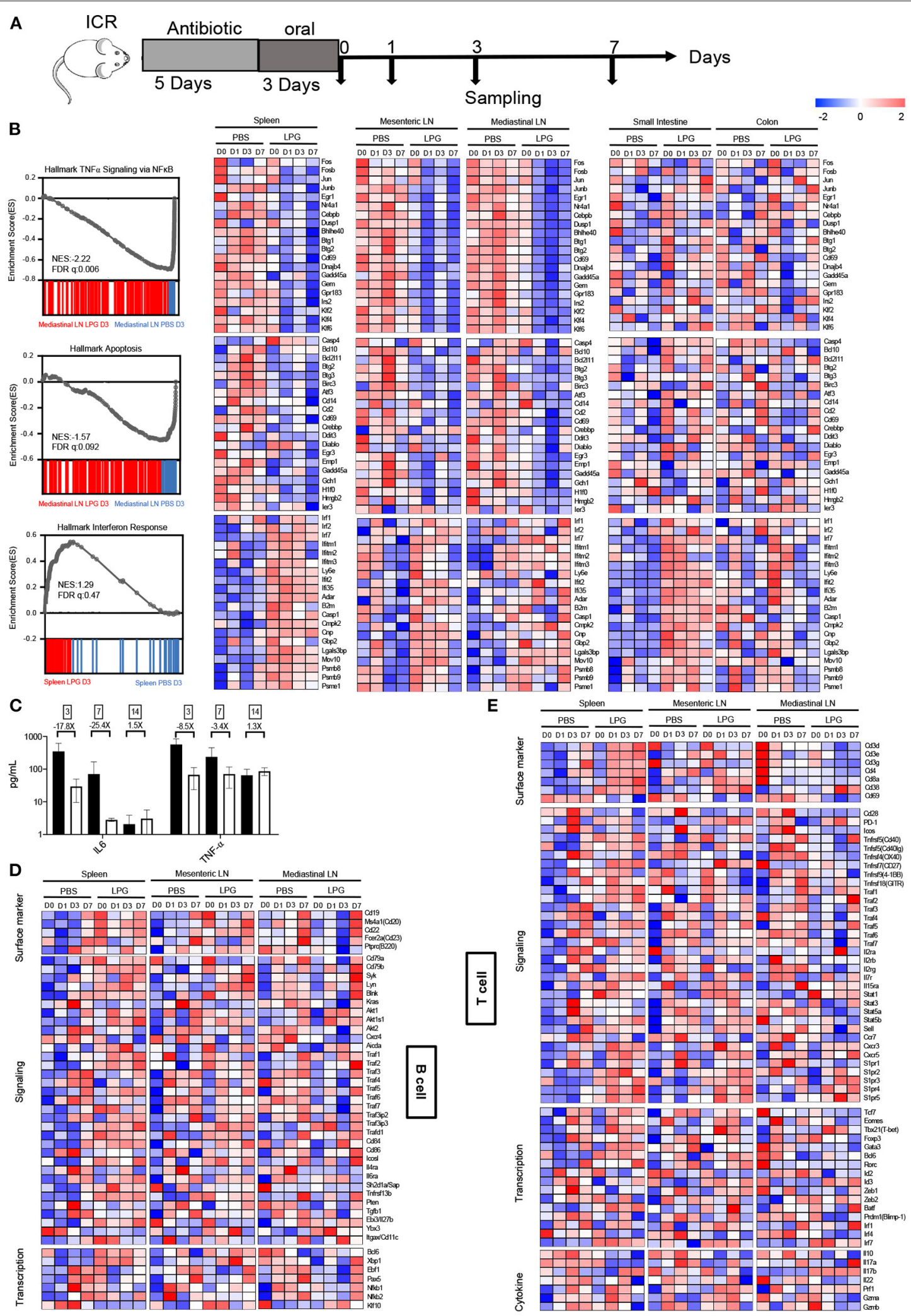

FIGURE 3 | Oral LPG administration down-regulates inflammation and apoptosis but promotes IFN signaling in mouse lymphoid tissues. Experimental schedule (A). ICR mice ( $n=20$ /group) were treated as described in the Methods, and different tissues were collected for RNA sequencing. GSEA revealed inhibition of TNF- $\alpha$ signaling (top) and apoptosis (middle) gene sets in lymphoid tissues in the LPG group compared with the PBS group, and enhancement of IFN response 
FIGURE 3 | (bottom) gene sets in lymphoid and small intestine tissues in the LPG group compared with the PBS group (B). IL-6 and TNF- $\alpha$ in BAL were quantified from mice sacrificed on day 3, 7, and 14 (C). Heatmap shows log2 RPKM values of a selection of genes associated with B cells (D) and T cells (E) from the spleen and small intestine. BAL, bronchoalveolar lavage; ES, enrichment score; FDR-q, false discovery rate q value; NES, normalized enrichment score.

should be implemented. Importantly, both clinical trial and realworld data have shown that neutralization titers against SARSCoV-2 (i.e., nAb values after adjustment with convalescent sera titers) correlate with protective efficacy (22), and breakthrough SARS-CoV-2 infection mainly occurs in subjects who receive vaccines with low nAb titers (23). For example, the regimen of rAd26-S plus rAd5-S elicited a 2-3-fold increase in $\mathrm{nAb}$ titers in comparison with ChAdOx1 nCoV-19 or Ad26.COV2.S. Correspondingly, the former conferred a 1.5 -fold increase in protective efficacy, suggesting that a $2-3$-fold difference in $\mathrm{nAb}$ titers is likely to significantly improve protection in the real world. It is therefore critical that licensed vaccines mount high, long-lasting $\mathrm{nAb}$ responses.

Here we demonstrated that the unique probiotic strain LBG boosted humoral responses against SARS-CoV-2 and stabilized $\mathrm{RBD}$-specific T-cell responses in mice when given immediately after vaccination. We observed a $>2$-fold increase of $n A b$ titers in LPG group sera and a $>8$-fold increase of nAb titers in LPG group BAL, which is more important than the increase in sera. The LPG group also exhibited enhanced T-cell responses, and as evidenced in Figure 1, LPG stabilized the memory responses for a prolonged time period. In an influenza vaccine clinical trial, administration of probiotic bacteria improved the proportion of nature killer cells and T-helper and T-cytotoxic cells. The greater immune response observed in the probiotic group in comparison with the placebo group correlated with a lower incidence of influenza-like illness in the 5 months of follow-up (24). Therefore, LGP may function as an adjuvant to enhance innate and adaptive immunity, thereby enhancing the protective efficacy and prolonging the protection from SARS-CoV-2 vaccination as observed for influenza and polio vaccination $(4,24-26)$.

Transcriptional data revealed that LPG alone could downregulate inflammatory responses and the apoptotic pathway and up-regulate IFN responses in the absence of vaccination. As down-regulation of TNF- $\alpha$ and apoptosis pathways largely affects immune cells, oral LPG administration is likely to heavily imprint immune cell activation and differentiation to enhance immune cell survival and memory pool formation. Up-regulation of the IFN response pathway could facilitate the clearance of any local tissue pathogens and promote immune responses. All these observations support our hypothesis that LPG boosted and prolonged SARS-CoV-2 immune responses. Furthermore, LPG is capable of affecting gut-spleen and gut-lung immune regulatory axes to overcome the barrier between mucosal and systemic compartments and mobilize immune responses (27).

Several B cell function-associated genes such as $A K T 1 / 2$, $A I C D A$, and $E B I 3$ were up-regulated in the LPG group. It is known that antigen recognition by $\mathrm{BCR}$ initiates the $\mathrm{BCR}$ signaling cascade by phosphorylation of $C D 79 A$ and $C D 79 B$ (28), which engages the tyrosine kinases $S Y K$ and $L Y N$ and the adapter protein $B L N K$. B cell-intrinsic $A K T 1 / 2$ function is required for germinal center (GC) formation, maintenance, and plasma cell differentiation (29). AICDA is associated with somatic hypermutation and class switching (15). EBI3 can promote B cell activation, and CD11c+ (ITGAX gene) B cells are mainly memory B cells $(30,31)$. All the factors mentioned above were upregulated in the LPG group, suggesting that LPG could improve $\mathrm{B}$ cell activation and differentiation to memory B cells.

Three signals are associated with $\mathrm{T}$ cell activation: signal 1 "antigenic stimulation," signal 2 "co-stimulatory signals through CD28 or 4-1BB," and signal 3 "cytokines of IL-12, IL-21 or IFN-alpha." In the current study, representative T-cell co-signal molecules of the CD28-B7, TNFSF/TNFRSF, and TRAF families $(32,33)$ were upregulated in the LPG group, as were survivalrelated cytokine genes (IL-2R, IL-7R, IL-15R, and STAT1/3/5) were up-regulated LPG group in this study. IL-2 is perhaps the earliest cytokine secreted by $\mathrm{T}$ cells following $\mathrm{T}$ cell receptor (TCR) stimulation (34), and it is important in the initiation of Th2 cell differentiation (35). It is well known that IL2 can induce the proliferation and survival of TCR-activated human and mouse $\mathrm{T}$ cells (36) and is required for sustained expansion of T cell populations (37). IL-7 seems to be the most important $\gamma_{c}$ family cytokine for regulating naïve and memory $\mathrm{T}$ cell homeostasis (38), while IL-15 is an important homeostatic cytokine with preferential activity for memory CD8+ T cells $(39,40)$. These data indicate that LPG could facilitate $\mathrm{T}$ cell survival and their differentiation into memory $\mathrm{T}$ cells.

Immune cell migration increases interaction between cells and is critical for the initiation of immune responses. In our study, the decrease of CCR7 and increase of S1PR family in the LPG group in the spleen promoted $\mathrm{T}$ cell emigration from lymph tissues. It has been speculated that emigration of CD8+ effector $\mathrm{T}$ cells from the T-zone may represent an important mechanism protecting professional antigen-presenting cells (APCs) against cytotoxic $\mathrm{T}$ cell attacks and preventing a premature decline in immune responses $(41,42)$, which may explain the prolonged T-cell specific response in the LPG group.

Overall, these transcriptional data demonstrated that oral administration of LPG is capable of enhancing gene expression associated with $\mathrm{T}$ cell and $\mathrm{B}$ cell activation, proliferation, survival, differentiation to memory cells, and migration. It is therefore rationalized that if co-administrated with vaccination, for example SARS-CoV-2 vaccine, $\mathrm{LPG}$ will enhance both immunogen-specific $\mathrm{T}$ and $\mathrm{B}$ cell responses, facilitate their mobilization among mucosal and systemic immune compartments, improve and prolong their memory responses.

A growing body of clinical data suggests that a cytokine storm is associated with COVID-19 severity and is a crucial cause of mortality $(43,44)$. IL- 6 and TNF- $\alpha$ are both key proinflammatory cytokines, and their suppression may attenuate disease severity. Notably, one of the proposed predisposing factors for a cytokine storm is a low level of type I IFN (45). As described above, we observed a $\sim 20$-fold reduction of IL-6 and 3-8-fold decrease of TNF- $\alpha$ on day 3 and 7, respectively, in the BAL of the LPG group compared with the PBS group. The IFN response pathway was up-regulated in 
both local tissue and immune cells, so oral LPG is likely to attenuate respiratory inflammation and help contain SARS-CoV2 replication, thereby ameliorating clinical manifestations and facilitate patient recovery.

In conclusion, our results demonstrated that LPG is able to boost vaccine-induced effective and memory immune responses by enhancing IFN signaling and suppressing apoptotic and inflammatory pathways. Co-administration of the SARS-CoV-2 vaccine and LPG show great potential to improve COVID-19 vaccination efficacy and contribute to the containment of the pandemic. Routine administration of LPG is likely to enhance the host innate immune responses to combat SARS-CoV-2.

\section{DATA AVAILABILITY STATEMENT}

The original contributions presented in the study are publicly available. This data can be found in the NCBI SRA database (accession numbers: 10520442, 10526585, 10614070).

\section{ETHICS STATEMENT}

The animal study was reviewed and approved by Institutional Animal Care and Use Committee (IACUC) of Shanghai Public Health Clinical Center (Shanghai, China).

\section{AUTHOR CONTRIBUTIONS}

JGX conceived and designed the experiments. JQX, ZHR, and KLC designed and implemented the SARS-CoV-2 related experiments. XL, JL, DJ, TY, and LL prepared and carried out experiments. LZ and KLC analyzed the transcriptional data. XW assisted animal study and ELISA. LD performed neutralization antibody titers. All authors contributed to the article and approved the submitted version.

\section{FUNDING}

This work was supported by National Key R and D Program of China (2019YFC1200501 and 2019 YFC 1200505), National Science and Technology Major Project of China (2018

\section{REFERENCES}

1. The COVID-19 vaccine race-weekly update. Available online at: http://www. gavi.org/vaccineswork/covid-19-vaccine-race

2. Haft JW, Atluri P, Ailawadi G, Engelman DT, Grant MC, Hassan A, et al. The COVID-19 exit strategy-why we need to aim low. Ann Thorac Surg. (2020) 110:697-700. doi: 10.1016/j.athoracsur.2020.04.003

3. Hodgson SH, Mansatta K, Mallett G, Harris V, Emary KRW, Pollard AJ. What defines an efficacious COVID-19 vaccine? A review of the challenges assessing the clinical efficacy of vaccines against SARS-CoV-2. Lancet Infect Dis. (2020) 21:e26-e35. doi: 10.1016/S1473-3099(20)30773-8

4. Gill H, Prasad J. Probiotics, immunomodulation, and health benefits. Adv Exp Med Biol. (2008) 606:423-54. doi: 10.1007/978-0-387-74087-4_17

5. Dosoky NS, Chen Z, Guo Y, McMillan C, Flynn CR, Davies SS. Twoweek administration of engineered Escherichia coli establishes persistent resistance to diet-induced obesity even without antibiotic pre-treatment.
Zx10712001-018), Research Units of Discovery of Unknown Bacteria and Function (2018RU010) to JGX, and Shanghai Municipal Science and Technology Commission (21S11903600), National Natural Science Foundation of China (81672018) to JQX.

\section{ACKNOWLEDGMENTS}

We thank the members of the JQX and JGX laboratory for advice and assistance during the study.

\section{SUPPLEMENTARY MATERIAL}

The Supplementary Material for this article can be found online at: https://www.frontiersin.org/articles/10.3389/fnut.2021. 789242/full\#supplementary-material

Supplementary Figure 1 | Lactobacillus plantarum GUANKE (LPG) genome analysis. The phylogenetic tree of $L$. plantarum (maximum-likelihood tree estimated using IQtree v.1.6.9) (A). The phylogeny was alignment based on the SNP of concatenated core genes with the recombination region removed. The GUANKE strain in our study is marked in red. Genome comparison of $L$. plantarum GUANKE with L. plantarum str. J26 (B). The inner second and third circles show the GC content and C/G skew of GUANKE. The outer circle shows the distribution and similarity of all ORFs in J26 compared to GUANKE with the missing genome annotated.

Supplementary Figure 2 | Oral administration of LPG 6 months after immunization did not change the total lgG titer in mice. Sera were collected post intragastric administration for total IgG assessment by ELISA (A), "Index" values are shown (B), which represents the ratios of titers of binding antibodies at indicated time-points to their counterparts at baseline and reflects the adjusted relative values after the removal of differences at baseline.

Supplementary Figure 3 | Oral LPG administration immediately after immunization stabilizes cellular immune responses in mice. Assessments of RBD-specific T cell responses. Splenocytes (A) and BAL cells (B) were isolated at day 3,7 , and 14 post intragastric administration and in vitro stimulated with 13 peptide pools (15-mer with 11 overlapped amino acids) covering the entire RBD sequence. The resulting IFN- $\gamma$-secreting cells were quantified by ELISpot. ELISpot counts are expressed as mean \pm s.e.m. Mann-Whitney tests were performed to analyze differences between experimental groups. ${ }^{* *} P<0.01$ and ns, not significant.

Supplementary Figure 4 | Hierarchical cluster analysis of $T$ and $B$ cell profiles from the spleen, mesenteric lymph node, mediastinal lymph node, small intestine, and colon. Heatmap with hierarchical clustering analysis including all groups subjected to RNA-sequencing of the T cell- and B cell-associated genes, with relative expression of log2 RPKM.
Appl Microbiol Biotechnol. (2019) 103:6711-23. doi: 10.1007/s00253-01909958-x

6. Wan J, Xing S, Ding L, Wang Y, Gu C, Wu Y, et al. Human-IgG-Neutralizing monoclonal antibodies block the SARS-CoV-2 infection. Cell Rep. (2020) 32:107918. doi: 10.1016/j.celrep.2020.107918

7. Qiu C, Huang Y, Zhang A, Tian D, Wan Y, Zhang X, et al. Safe pseudovirusbased assay for neutralization antibodies against influenza $\mathrm{A}(\mathrm{H} 7 \mathrm{~N} 9)$ virus. Emerg Infect Dis. (2013) 19:1685-7. doi: 10.3201/eid1910.130728

8. Lin J, Xu J. Development of genetic-engineered-microbiological vaccine for Shigella species (Master thesis). Chinese Academy of Preventive Medicine, Beijing, China (1998).

9. Zhang Z, Man C, Sun L, Yang X, Li M, Zhang W, et al. Short communication: complete genome sequence of Lactobacillus plantarum J26, a probiotic strain with immunomodulatory activity. J Dairy Sci. (2019) 102:10838-44. doi: 10.3168/jds.2019-16593 
10. Canzoneri R, Naipauer J, Stedile $M$, Peña AR, Lacunza E. Identification of an AP1-ZFP36 regulatory network associated with breast cancer prognosis. J Mammary Gland Biol Neoplasia. (2020) 25:163-72. doi: 10.1007/s10911-020-09448-1

11. Du Y, Liu P, Zang W. BTG3 upregulation induces cell apoptosis and suppresses invasion in esophageal adenocarcinoma. Mol Cell Biochem. (2015) 404:318. doi: 10.1007/s11010-015-2363-9

12. Mao B, Zhang Z, Wang G. BTG2 : a rising star of tumor suppressors (Review). Int J Oncol. (2015) 16:459-64. doi: 10.3892/ijo.2014.2765

13. Diamond MS, Farzan M. The broad-spectrum antiviral functions of IFIT and IFITM proteins. Nat Rev Immunol. (2013) 13:46-57. doi: 10.1038/nri3344

14. Laidlaw BJ, Cyster JG. Transcriptional regulation of memory B cell differentiation. Nat Rev Immunol. (2021) 21:20920. doi: 10.1038/s41577-020-00446-2

15. Xu Z, Pone EJ, Al-Qahtani A, Park SR, Zan H, Casali P. Regulation of aicda expression and AID activity: Relevance to somatic hypermutation and class switch DNA recombination. Crit Rev Immunol. (2007) 27:36797. doi: 10.1615/critrevimmunol.v27.i4.60

16. Shaffer AL, Shapiro-Shelef M, Iwakoshi NN, Lee AH, Qian SB, Zhao H, et al. XBP1, downstream of Blimp-1, expands the secretory apparatus and other organelles, and increases protein synthesis in plasma cell differentiation. Immunity. (2004) 21:81-93. doi: 10.1016/j.immuni.2004.06.010

17. Luckheeram RV, Zhou R, Verma AD, Xia B. CD4 + T cells: differentiation and functions. Clin Dev Immunol. (2012) 2012:925135. doi: 10.1155/2012/925135

18. Xin G, Schauder DM, Lainez B, Weinstein JS, Dai Z, Chen Y, et al. A critical role of IL-21-induced BATF in sustaining CD8-T-cell-mediated chronic viral control. Cell Rep. (2015) 13:1118-24. doi: 10.1016/j.celrep.2015.09.069

19. Kuwabara T, Ishikawa F, Kondo M, Kakiuchi T. The role of IL-17 and related cytokines in inflammatory autoimmune diseases. Mediators Inflamm. (2017) 2017:3908061. doi: 10.1155/2017/3908061

20. Tian T, Wan J, Han Y, Liu H, Gao F, Pan Y, et al. A comprehensive survey of immune cytolytic activity-associated gene co-expression networks across 17 tumor and normal tissue types. Cancers (Basel). (2018) 10:307. doi: 10.3390/cancers10090307

21. Anderson RM, Vegvari C, Truscott J, Collyer BS. Challenges in creating herd immunity to SARS-CoV-2 infection by mass vaccination. Lancet. (2020) 396:1614-6. doi: 10.1016/S0140-6736(20)32318-7

22. Khoury DS, Cromer D, Reynaldi A, Schlub TE, Wheatley AK, Juno JA, et al. Neutralizing antibody levels are highly predictive of immune protection from symptomatic SARS-CoV-2 infection. Nat Med. (2021) 27:1205-11. doi: 10.1038/s41591-021-01377-8

23. Bergwerk M, Gonen T, Lustig Y, Amit S, Lipsitch M, Cohen C, et al. Covid19 breakthrough infections in vaccinated health care workers. $N$ Engl J Med. (2021) 385:1474-84. doi: 10.1056/nejmoa2109072

24. Olivares M, Díaz-Ropero MP, Sierra S, Lara-Villoslada F, Fonollá J, Navas M, et al. Oral intake of Lactobacillus fermentum CECT5716 enhances the effects of influenza vaccination. Nutrition. (2007) 23:25460. doi: 10.1016/j.nut.2007.01.004

25. Nova E, Wärnberg J, Gómez-Martínez S, Díaz LE, Romeo J, Marcos A. Immunomodulatory effects of probiotics in different stages of life. Br J Nutr. (2007) 98:90-5. doi: 10.1017/S0007114507832983

26. Bosch M, Méndez M, Pérez M, Farran A, Fuentes MC, Cuñé J. Lactobacillus plantarum CECT7315 and CECT7316 stimulate immunoglobulin production after influenza vaccination in elderly. Nutr Hosp. (2012) 27:504-9. doi: 10.3305/nh.2012.27.2.5519

27. Anand S, Mande SS. Diet, microbiota and gut-lung connection. (2018) Front Microbiol. 9:2147. doi: 10.3389/fmicb.2018.02147

28. Liu J, Wang Y, Min Q, Xiong E, Heyman B, Wang JY. Regulation of Humoral Immune Responses and B Cell Tolerance by the IgM Fc Receptor (Fc $\mu R)$. Adv Exp Med Biol. (2020) 1254:75-86. doi: 10.1007/978-981-15-3532-1_7

29. Zhu Z, Shukla A, Ramezani-Rad P, Apgar JR, Rickert RC. The AKT isoforms 1 and 2 drive $B$ cell fate decisions during the germinal center response. Life Sci Alliance. (2019) 2:1-19. doi: 10.26508/lsa.201900506

30. Ma N, Fang Y, Xu R, Zhai B, Hou C, Wang X, et al. Ebi3 promotes Tand B-cell division and differentiation via STAT3. Mol Immunol. (2019) 107:61-70. doi: 10.1016/j.molimm.2019.01.009

31. Golinski ML, Demeules M, Derambure C, Riou G, Maho-Vaillant M, Boyer $\mathrm{O}$, et al. CD11c+ $\mathrm{B}$ cells are mainly memory cells, precursors of antibody secreting cells in healthy donors. Front Immunol. (2020) 11:32. doi: 10.3389/fimmu.2020.00032

32. Ward-Kavanagh LK, Lin WW, Šedý JR, Ware CF. The TNF receptor superfamily in co-stimulating and co-inhibitory responses. Immunity. (2016) 44:1005-19. doi: 10.1016/j.immuni.2016.04.019

33. Watts TH. TNF/TNFR family members in costimulation of $\mathrm{T}$ cell responses. Annu Rev Immunol. (2005) 23:2368. doi: 10.1146/annurev.immunol.23.021704.115839

34. Sojka DK, Bruniquel D, Schwartz RH, Singh NJ. IL-2 secretion by CD4 + T cells in vivo is rapid, transient, and influenced by TCR-specific competition. J Immunol. (2004) 172:6136-43. doi: 10.4049/jimmunol.172.10.6136

35. Cote-Sierra J, Foucras G, Guo L, Chiodetti L, Young HA, Hu-Li J, et al. Interleukin 2 plays a central role in Th2 differentiation. Proc Natl Acad Sci USA. (2004) 101:3880-5. doi: 10.1073/pnas.0400339101

36. Kim HP, Imbert J, Leonard WJ. Both integrated and differential regulation of components of the IL-2/IL-2 receptor system. Cytokine Growth Factor Rev. (2006) 17:349-66. doi: 10.1016/j.cytogfr.2006.07.003

37. D'Souza WN, Lefrançois L. IL-2 is not required for the initiation of CD8 T cell cycling but sustains expansion. J Immunol. (2003) 171:572735. doi: 10.4049/jimmunol.171.11.5727

38. Seddon B, Tomlinson P, Zamoyska R. Interleukin 7 and $\mathrm{T}$ cell receptor signals regulate homeostasis of CD4 memory cells. Nat Immunol. (2003) 4:680-6. doi: 10.1038/ni946

39. Berard M, Brandt K, Bulfone-Paus S, Tough DF. IL-15 promotes the survival of naive and memory phenotype CD8 + T cells. J Immunol. (2003) 171:2170. doi: 10.4049/jimmunol.171.4.2170-a

40. Rochman Y, Spolski R, Leonard WJ. New insights into the regulation of $\mathrm{T}$ cells by $\gamma \mathrm{c}$ family cytokines. Nat Rev Immunol. (2010) 9:123. doi: $10.1038 /$ nri2580.New

41. Yan Y, Chen R, Wang X, Hu K, Huang L, Lu M, et al. CCL19 and CCR7 expression, signaling pathways, and adjuvant functions in viral infection and prevention. Front Cell Dev Biol. (2019) 7:1-13. doi: 10.3389/fcell.2019.00212

42. Choi H, Song H, Jung YW. The roles of CCR7 for the homing of memory cd8+ $\mathrm{t}$ cells into their survival niches. Immune Netw. (2020) 20:115. doi: 10.4110/in.2020.20.e20

43. Marcuzzi A, Melloni E, Zauli G, Romani A, Secchiero P, Maximova $\mathrm{N}$, et al. Autoinflammatory diseases and cytokine stormsimbalances of innate and adaptative immunity. Int J Mol Sci. (2021) 22:11241. doi: 10.3390/ijms222011241

44. Attiq A, Jin L, Afzal S, Ali M. The triumvirate of NF- $\kappa \mathrm{B}$, inflammation and cytokine storm in COVID-19. Int Immunopharmacol. (2021) 101(Pt B):108255. doi: 10.1016/j.intimp.2021.108255

45. Soy M, Keser G, Atagündüz P, Tabak F, Atagündüz I, Kayhan S. Cytokine storm in COVID-19: pathogenesis and overview of antiinflammatory agents used in treatment. Clin Rheumatol. (2020) 39:208594. doi: 10.1007/s10067-020-05190-5

Conflict of Interest: JGX, JQX, ZHR, and KLC filed patent describing the invention and use of the probiotic described in the manuscript.

The remaining authors declare that the research was conducted in the absence of any commercial or financial relationships that could be construed as a potential conflict of interest.

Publisher's Note: All claims expressed in this article are solely those of the authors and do not necessarily represent those of their affiliated organizations, or those of the publisher, the editors and the reviewers. Any product that may be evaluated in this article, or claim that may be made by its manufacturer, is not guaranteed or endorsed by the publisher.

Copyright (C) 2021 Xu, Ren, Cao, Li, Yang, Luo, Zhu, Wang, Ding, Liang, Jin, Yuan, $L i$ and $X u$. This is an open-access article distributed under the terms of the Creative Commons Attribution License (CC BY). The use, distribution or reproduction in other forums is permitted, provided the original author(s) and the copyright owner(s) are credited and that the original publication in this journal is cited, in accordance with accepted academic practice. No use, distribution or reproduction is permitted which does not comply with these terms. 